\title{
Classical Call-by-need and duality
}

\author{
Zena M. Ariola \& Hugo Herbelin \& Alexis Saurin \\ University of Oregon \& Laboratoire PPS, équipe $\pi r^{2}$, CNRS, INRIA \& Université \\ Paris Diderot \\ ariola@cs.uoregon.edu herbelin@inria.fr saurin@pps.jussieu.fr
}

\begin{abstract}
We study call-by-need from the point of view of the duality between call-by-name and call-by-value. We develop sequent-calculus style versions of call-by-need both in the minimal and classical case. As a result, we obtain a natural extension of call-by-need with control operators. This leads us to introduce a call-by-need $\lambda \mu$-calculus. Finally, by using the dualities principles of $\bar{\lambda} \mu \tilde{\mu}$-calculus, we show the existence of a new call-by-need calculus, which is distinct from call-by-name, call-byvalue and usual call-by-need theories ${ }^{1}$

Keywords: call-by-need, lazy evaluation, duality of computation, sequent calculus, $\lambda \mu$-calculus, classical logic, control, subtraction connective
\end{abstract}

\section{Introduction}

The theory of call-by-name $\lambda$-calculus [10/9] is easy to define. Given the syntax of $\lambda$-calculus $M::=x\|\lambda x . M\| M M$, the reduction semantics is entirely determined by the $\beta$-reduction rule $(\lambda x . M) N \rightarrow_{\beta} M[x \leftarrow N]$ in the sense that:

- for any closed term $M$, either $M$ is a value $\lambda x . N$ or $M$ is a $\beta$-redex and for all $M \rightarrow V$, there is standard path $M \mapsto V^{\prime}$ made only of $\beta$-redexes at the head, together with the property that $V^{\prime} \rightarrow V$ using internal $\beta$-reductions;

- the observational closure of $\beta$ induces a unique rule $\eta$ that fully captures observational equality over finite normal terms (so-called Böhm's theorem);

- the extension with control, typically done à la Parigot [30, is relatively easy to get by adding just two operational rules and an observational rule (though the raw version of Böhm's theorem fails [16[35]).

The theory of call-by-value $\lambda$-calculus, as initiated by Plotkin [31, has a similar property with respect to the $\beta_{v}$ rule (the argument of $\beta$ being restricted to a variable or a $\lambda x . M$ only), but the observational closure is noticeably more complex: it at least includes the rules unveiled by Moggi [27] as was shown by Sabry and Felleisen 34]. Extensions of standardization and Böhm theorem for call-by-value are more delicate than in call-by-name [25/33].

Comparatively, call-by-need $\lambda$-calculus, though at the core of implementation languages such as Haskell [17, is in a rudimentary state of development as the

${ }^{1}$ An extended version [7] is available at http://www.pps.jussieu.fr/ ${ }^{\text {saurin/ }}$ Publi/need_duality_long.pdf 
first approach to it as a proper calculus goes back to the 90's with the works of Ariola et al. 33 and Maraist et al. 26, for whom the concern was the characterization of standard weak-head reduction. Our paper is an attempt to raise the study of call-by-need to the same level of study as call-by-name, and in a slightly less extent call-by-value. In particular, we will address the question of adding control to call-by-need and the question of what is the dual of call-by-need along the lines of the duality between call-by-name and call-by-value [213612]. Callby-need is close to call-by-value in the sense that only values are substituted, but call-by-need is also close to call-by-name in the sense that only those terms that are bound to needed variables are evaluated. In particular, with respect to evaluation of pure closed terms, the call-by-name and call-by-need calculi are not distinguishable. In order to tackle the problem of developing a classical version of call-by-need, we first study how to formulate (minimal) call-by-need in the sequent calculus setting [23] (while current call-by-need calculi are based on natural deduction [32]). An advantage of a sequent calculus presentation of a calculus is that its extension to the classical case does not require the introduction of new rules but simply the extension of existing ones [8].

Curien and Herbelin [12] designed a calculus that provides an appealing computational interpretation of proofs in sequent calculus, while providing at the same time a syntactic duality between terms, i.e., producers, and evaluation contexts, i.e., consumers, and between the call-by-name and call-by-value reduction strategies. By giving priority to the producer one obtains call-by-value, whereas by giving priority to the consumer one obtains call-by-name. In this paper, we present how call-by-need fits in the duality of computation. Intuitively, call-by-need corresponds to focusing on the consumer to the point where the producer is needed. The focus goes then to the producer till a value is reached. At that point, the focus returns to the consumer. We call this calculus lazy call-by-value, it is developed in Section 2 and 3 . In addition to the properties of confluence and standardization, we show its correctness with respect to the call-by-name sequent calculus [12. In Section 4, we develop the natural deduction presentation of call-by-need. The reduction theory is contained in the one of Maraist et al. [26] and extends the one of Ariola et al. [3]. Interestingly, the sequent calculus has suggested an alternative standard reduction which consists of applying some axioms (i.e., lift and assoc) eagerly instead of lazily. In Section 5. we show that the natural deduction and sequent calculus call-by-need are in reduction correspondence. In Section 6, we extend the minimal sequent calculus call-by-need with control, in both sequent calculus and natural deduction form. The calculi still enjoy confluence and standardization. The sequent calculus presentation of call-by-need naturally leads to a dual call-by-need, which corresponds to focusing on the producer and going to the consumer on a need basis. We call this calculus lazy call-by-name. In Section 7 , we show how the dual call-by-need is obtained by dualizing the lazy call-by-value extended with the subtraction connective. We conclude and discuss our future work in Section 8. We start next with an overview of the duality of computation. 


\section{The duality of computation}

Curien and Herbelin [12] provided classical sequent calculus with a term assignment, which is called the $\bar{\lambda} \mu \tilde{\mu}$ calculus. In $\bar{\lambda} \mu \tilde{\mu}$ there are two dual syntactic categories: terms which produce values and contexts which consume values. The interaction between a producer $v$ and a consumer $e$ is rendered by a command written as $\langle v \| e\rangle$, which is the computational counterpart of a sequent calculus cut. Contexts can be seen as evaluation contexts, that is, commands with a hole, written as $\square$, standing for the term whose computation is to be done next: $\langle\square \| e\rangle$. Thus, a command $\langle v \| e\rangle$ can be seen as filling the hole of the evaluation context $e$ with $v$. Dually, terms can also be seen as commands with a context hole, standing for the context in which the term shall be computed. The duality of terms and contexts is also reflected at the variable level. One has two distinct sets of variables. The usual term variables $(x, y, \cdots)$ and the context variables $(\alpha, \beta, \cdots)$, which correspond to continuation variables. The set of terms, in addition to variables and lambda abstractions, contains a term of the form $\mu \alpha . c$, where $c$ is a command, after Parigot's $\lambda \mu$-calculus 30 . The $\mu$ construct corresponds to Felleisen's $\mathcal{C}$ control operator [192018]; one can read $\mu \alpha . c$ as $\mathcal{C}(\lambda \alpha . c)$. Whereas the $\mu$ construct allows one to give a name to a context, so as to invoke it later, the dual construct, named $\tilde{\mu}$, allows one to name terms. One can read $\tilde{\mu} x . c$ as let $x=\square$ in $c$. Given a context $e, v \cdot e$ is also a context, which corresponds to an applicative context of the form $e[\square v]$. The grammar of $\bar{\lambda} \mu \tilde{\mu}$ and its reduction theory are given below:

$$
\begin{aligned}
& c::=\langle v \| e\rangle \quad v::=x|\lambda x . v| \mu \alpha . c \quad e::=\alpha \mid \tilde{\mu} x . c \| v \cdot e \\
& \text { (в) }\langle\lambda x . v \| s \cdot e\rangle \rightarrow\langle s \| \tilde{\mu} x .\langle v \| e\rangle\rangle \\
& \text { ( } \mu) \quad\langle\mu \alpha . c \| e\rangle \rightarrow c[\alpha \leftarrow e] \\
& (\tilde{\mu}) \quad\left\langle v \| \tilde{\mu} x . c^{\prime}\right\rangle \quad \rightarrow c^{\prime}[x \leftarrow v]
\end{aligned}
$$

The reduction theory can be seen as consisting of structural reduction rules, $\mu$ and $\tilde{\mu}$, as well as logical reduction rules (here, only $\beta$, the rule corresponding to implication).

The calculus is not confluent due to a critical pair between $\mu$ and $\tilde{\mu}$ :

$$
\langle z \| \beta\rangle \leftarrow_{\mu}\langle\mu \alpha .\langle z \| \beta\rangle \| \tilde{\mu} x .\langle y \| \beta\rangle\rangle \rightarrow_{\tilde{\mu}}\langle y \| \beta\rangle
$$

To regain confluence one can impose a strategy on how to resolve the critical pair $\mu / \tilde{\mu}$. By giving priority to the $\tilde{\mu}$ rule one captures call-by-name, whereas by giving priority to the $\mu$ rule one captures call-by-value. More generally, one can describe various ways to specialize the pair $\mu / \tilde{\mu}$ as reduction rules parametrized by sets $\mathcal{E}$ and $\mathcal{V}$, which denote sets of contexts and terms, respectively:

$$
\begin{array}{ll}
\left(\mu_{\mathcal{E}}\right)\langle\mu \alpha . c \| e\rangle \rightarrow c[\alpha \leftarrow e] & \text { if } e \in \mathcal{E} \\
\left(\tilde{\mu}_{\mathcal{V}}\right)\left\langle v \| \tilde{\mu} x . c^{\prime}\right\rangle \rightarrow c^{\prime}[x \leftarrow v] & \text { if } v \in \mathcal{V}
\end{array}
$$

This presentation with parametric rules is inspired by the work of Ronchi and Paolini on parametric $\lambda$-calculus [33]. A strategy corresponds to specifying which 
contexts and terms can be duplicated or erased. For call-by-name, $\mathcal{E}$ and $\mathcal{V}$ are instantiated with the following sets $E_{n}$ and $V_{n}$ :

$$
E_{n}::=\alpha\left\|v \cdot E_{n} \quad V_{n}::=x \mid \lambda x . v\right\| \mu \alpha . c
$$

resulting in reduction rules that we will denote as $\mu_{n}$ and $\tilde{\mu}_{n}$, respectively. For call-by-value, the instantiations are $E_{v}$ and $V_{v}$ :

$$
E_{v}::=\alpha\left|v \cdot E_{v}\right| \tilde{\mu} x . c \quad V_{v}::=x \mid \lambda x . v
$$

resulting in reduction rules that we will denote as $\mu_{v}$ and $\tilde{\mu}_{v}$, respectively. In call-by-name $\left(i . e .\right.$, with $\left.\mu_{n} / \tilde{\mu}_{n}\right)$ every term is substitutable for a term variable, while only specific contexts can be substituted for a context variable. Dually, callby-value (i.e., with $\mu_{v} / \tilde{\mu}_{v}$ ) restricts what is substituted for a term variable, but does not impose restrictions on substitution of contexts. Notice also that full non-deterministic $\bar{\lambda} \mu \tilde{\mu}$ corresponds to choosing $\mu_{v}$ together with $\tilde{\mu}_{n}$. Call-byneed $\bar{\lambda} \mu \tilde{\mu}$-calculus will be defined with respect to another choice of parameters.

Curien and Herbelin also developed a subcalculus of $\bar{\lambda} \mu \tilde{\mu}$ called $\bar{\lambda} \mu \tilde{\mu}_{T}$ (after Danos et al's LKT [13[14]), which restricts the syntax of legal contexts. This captures the intuition that according to the call-by-name continuation passing style, the continuation follows a specific pattern. The syntax of $\bar{\lambda} \mu \tilde{\mu}_{T}$ becomes:

$$
c::=\langle v \| e\rangle \quad v::=V_{n} \quad e::=\tilde{\mu} x . c \| E_{n}
$$

Notice that whereas $v \cdot \tilde{\mu} x . c$ is a legal context in $\bar{\lambda} \mu \tilde{\mu}$, it is not legal in $\bar{\lambda} \mu \tilde{\mu}_{T}$. The reduction theory of $\bar{\lambda} \mu \tilde{\mu}_{T}$ consists of $\beta, \mu_{n}$ and $\tilde{\mu}_{n}$.

In addition to the instantiations of the structural rules $\mu_{\mathcal{E}}$ and $\tilde{\mu}_{\mathcal{V}}$, the calculi developed in the rest of the paper will contain rules for evaluating connectives. We will only consider implication, except in Section 7 where subtraction will also be added. We will also consider the following extensionality rules:

$$
\begin{array}{ll}
\left(\eta_{\mu}^{\mathcal{V}}\right) \mu \alpha .\langle v \| \alpha\rangle \rightarrow v & v \in \mathcal{V} \text { and } \alpha \text { is not free in } v \\
\left(\eta_{\tilde{\tilde{\mu}}}\right) \tilde{\mu} x .\langle x \| e\rangle \rightarrow e & e \in \mathcal{E} \text { and } x \text { is not free in } e
\end{array}
$$

\section{Call-by-need and duality}

As we did for call-by-name and call-by-value, we have to specify the parametric sets used for call-by-need, that is, which terms and contexts can be substituted for term and context variables. Since call-by-need avoids duplication of work, it is natural to restrict the set $\mathcal{V}$ to $V_{v}$, thus allowing substitution of variables and lambda abstractions only. One should allow the reduction of $\langle\mu \alpha .\langle I \| I \cdot \alpha\rangle \| v \cdot \alpha\rangle$ ( $I$ stands for $\lambda x \cdot x$ ) to $\langle I \| I \cdot v \cdot \alpha\rangle$ since the applicative redex is needed in order to continue the computation. This implies that $E_{n}$ should be part of $\mathcal{E}$. That however is not enough. One would also want to reduce $\langle\mu \alpha .\langle I \| I \cdot \alpha\rangle \| \tilde{\mu} x .\langle x \| \alpha\rangle\rangle$ to $\langle I \| I \cdot \tilde{\mu} x .\langle x \| \alpha\rangle\rangle$. This however does not imply that $\tilde{\mu} x . c$ should be part of $\mathcal{E}$ since that would unveil an unwanted redex, such as in $\langle\mu \alpha .\langle I \| I \cdot \alpha\rangle \| \tilde{\mu} x \cdot\langle z \| \alpha\rangle\rangle$. The only time we want to allow a change of focus from the consumer to the 
producer is when the producer is needed, which means that the variable bound to the producer occurs in the hole of a context; $x$ is needed in $\left\langle x \| E_{n}\right\rangle$ but it is not needed in $\langle x \| \tilde{\mu} y \cdot\langle z \| x \cdot y\rangle\rangle$. This notion will still not capture a situation such as $\left\langle\mu \alpha .\langle I \| I \cdot \alpha\rangle \| \tilde{\mu} x \cdot\left\langle v_{1} \| \tilde{\mu} y \cdot\left\langle x \| E_{n}\right\rangle\right\rangle\right\rangle$, since the needed variable is buried under the binding for $y$. This motivates the introduction of the notion of a call-by-need meta-context, which is simply a hole surrounded by $\tilde{\mu}$-bindings:

$$
C_{l}^{\tilde{\mu}}::=\square \mid\left\langle\mu \alpha \cdot c \| \tilde{\mu} z \cdot C_{l}^{\tilde{\mu}}\right\rangle
$$

A variable $x$ is needed in a command $c$, if $c$ is of the form $C_{l}^{\tilde{\mu}}\left[\left\langle x \| E_{n}\right\rangle\right]$.

We have so far determined that $\mathcal{E}$ contains the call-by-name applicative contexts and contexts of the form $\tilde{\mu} x . C_{l}^{\tilde{\mu}}\left[\left\langle x \| E_{n}\right\rangle\right]$. This would allow the reduction of $\langle\mu \alpha .\langle I \| I \cdot \alpha\rangle \| \tilde{\mu} f \cdot\langle f \| f \cdot \alpha\rangle\rangle$ to $\langle I \| I \cdot \tilde{\mu} f \cdot\langle f \| f \cdot \alpha\rangle\rangle$. The problem is that the callby-name applicative context considered so far does not contain a $\tilde{\mu}$. This is necessary to capture sharing. For example, in the above term $\langle I \| I \cdot \tilde{\mu} f \cdot\langle f \| f \cdot \alpha\rangle\rangle$, the $\tilde{\mu} f$ captures the sharing of $I I$. We need however to be careful about which $\tilde{\mu}$ we allow in the notion of applicative context. For example, we should disallow contexts such as $I \cdot \tilde{\mu} f \cdot\langle z \| f \cdot \alpha\rangle$ since they might cause unwanted computation. Indeed, in the following reduction the application of $I$ to $I$ is computed while it is not needed to derive the result:

$$
\langle I \| I \cdot \tilde{\mu} f \cdot\langle z \| f \cdot \alpha\rangle\rangle \rightarrow_{\beta}\langle I \| \tilde{\mu} x .\langle x \| \tilde{\mu} f \cdot\langle z \| f \cdot \alpha\rangle\rangle\rangle \rightarrow_{\tilde{\mu}_{v}}\langle I \| \tilde{\mu} f \cdot\langle z \| f \cdot \alpha\rangle\rangle \rightarrow_{\tilde{\mu}_{v}}\langle z \| I \cdot \alpha\rangle .
$$

This implies that a context $\tilde{\mu} x . c$ is allowed in an applicative context only if $c$ demands $x$.

We are ready to instantiate the structural and extensional rules; $\mathcal{V}$ and $\mathcal{E}$ are instantiated as follows, resulting in reduction rules denoted as $\mu_{l}, \tilde{\mu}_{v}$ and $\eta_{\mu}^{v}$ :

$$
V_{v}::=x\left\|\lambda x . v \quad E_{l}::=F\right\| \tilde{\mu} x . C_{l}^{\tilde{\mu}}[\langle x \| F\rangle] \quad \text { with } \quad F::=\alpha \| v \cdot E_{l}
$$

\section{Minimal call-by-need in sequent form $\left(\boldsymbol{\lambda}_{m l v}\right)$}

A classical sequent calculus naturally provides a notion of control. However, one can restrict the calculus to be control-free by limiting the set of continuation variables to a single variable, conventionally written $\star$, which is linearly used. This corresponds to the restriction to minimal logic [1]. We introduce next the lazy call-by-value calculus, $\lambda_{m l v}$.

Definition 1. The syntax of $\lambda_{m l v}$ is defined as follows:

$$
\begin{array}{ll}
\text { command } & c::=\langle v \| e\rangle \\
\text { meta-context } & C::=\square \mid\langle\mu \star . c \| \tilde{\mu} z . C\rangle \\
\text { term } & v::=x \mid \lambda x . v \| \mu \star . c \\
\text { applicative context } F::=\star \mid v \cdot E \\
\text { linear context } & E::=F \mid \tilde{\mu} x . C[\langle x \| F\rangle] \\
\text { context } & e::=E \mid \tilde{\mu} x . c
\end{array}
$$


The reduction of $\lambda_{m l v}$, written as $\rightarrow_{m l v}$, denotes the compatible closure of $\beta$, $\mu_{l}, \tilde{\mu}_{v}$ and $\eta_{\mu}^{v}$; the relation $\rightarrow \rightarrow_{m l v}$ denotes the reflexive and transitive closure of $\rightarrow_{\text {mlv }}$. The notion of weak head standard reduction is defined as:

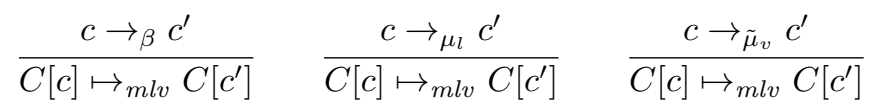

The notation $\mapsto_{\text {mlv }}$ stands for the reflexive and transitive closure of $\mapsto_{m l v}$. $A$ weak head normal form (whnf) is a command $c$ such that for no $c^{\prime}, c \mapsto_{m l v} c^{\prime}$.

Notice how in the lazy call-by-value calculus, the standard redex does not necessarily occur at the top level, in $\left\langle v_{1} \| \tilde{\mu} x_{1} \cdot\left\langle v_{2} \| \tilde{\mu} x_{2} \cdot\langle\lambda x \cdot v \| s \cdot \star\rangle\right\rangle\right\rangle$, the standard redex is buried under the bindings for $x_{1}$ and $x_{2}$, which is why the standard reduction refers to the meta-context. This however can be solved simply by going to a calculus with explicit substitutions, which would correspond to the abstract machine given in [7]. Note that in a term of the form $\langle\lambda z \cdot v \| \tilde{\mu} x .\langle x \| \tilde{\mu} y .\langle y \| \star\rangle\rangle\rangle$, the substitution for $y$ is not the standard redex, and in

$$
\langle\mu \star .\langle I \| I \cdot \star\rangle \| \tilde{\mu} x \cdot \underline{\langle x \| \tilde{\mu} y \cdot\langle y \| \star\rangle\rangle\rangle \quad \underline{\langle\mu \star} \cdot\langle V \| \tilde{\mu} y \cdot\langle y \| \star\rangle\rangle \| \tilde{\mu} x \cdot\langle x \| \star\rangle\rangle}
$$

the standard redex is the underlined one. The $\eta_{\mu}^{v}$ rule is not needed for standard reduction. The $\eta_{\mu}^{v}$ rule turns a computation into a value, allowing for example $\langle\mu \star .\langle V \| \star\rangle \| \tilde{\mu} x .\langle y \| x \cdot \star\rangle\rangle \rightarrow\langle V \| \tilde{\mu} x \cdot\langle y \| x \cdot \star\rangle\rangle \rightarrow\langle y \| V \cdot \star\rangle$, which is not a standard reduction; in fact, the starting term is already in whnf.

Proposition 1. $\rightarrow_{m l v}$ is confluent.

Remark 1 In $\lambda_{m l v}$ the duplicated redexes are all disjoint. This was not the situation in $\lambda_{\text {need }}$ [26], where the assoc rule could have duplicated a lift redex. This does not happen in $\lambda_{m l v}$ because the contexts are moved all at once, as described in the example below, which mimics the situation in $\lambda_{\text {need }}$.

$$
\begin{aligned}
\langle\mu \star\langle\mu \star \cdot\langle z \| \tilde{\mu} y \cdot\langle y \| \star\rangle\rangle \| \tilde{\mu} x \cdot\langle x \| \star\rangle\rangle \| N \cdot \star\rangle & \rightarrow \mu_{n}\langle\mu \star \cdot\langle z \| \tilde{\mu} y \cdot\langle y \| \tilde{\mu} x \cdot\langle x \| \star\rangle\rangle\rangle \| N \cdot \star\rangle \\
\downarrow_{\mu_{n}} & \downarrow_{\mu_{n}} \\
\langle\mu \star \cdot\langle z \| \tilde{\mu} y \cdot\langle y \| \star\rangle\rangle \| \tilde{\mu} x \cdot\langle x \| N \cdot \star\rangle\rangle & \rightarrow \mu_{n}\langle z \| \tilde{\mu} y \cdot\langle y \| \tilde{\mu} x \cdot\langle x \| N \cdot \star\rangle\rangle\rangle
\end{aligned}
$$

The needed constraint breaks the property that commands in weak head normal form are of the form $\langle x \| E\rangle$ or $\langle\lambda x . v \| \star\rangle$ (a property that holds for $\bar{\lambda} \mu \tilde{\mu}$ in call-by-name or call-by-value).

Definition 2. Let $\boldsymbol{x}$ be a sequence of variables. $c_{\boldsymbol{x}}$ is defined by the grammar:

$$
c_{\boldsymbol{x}}::=\left\langle\mu \star .\|\| \tilde{\mu} y . c_{y \boldsymbol{x}}\right\rangle|\langle\lambda x . v \| \star\rangle|\langle z \| F\rangle \quad z \notin \boldsymbol{x} .
$$

Proposition 2. A command $c$ is in weak head normal form iff it is in $c_{\epsilon}$, where $\epsilon$ denotes the empty sequence of variables.

Note that in $c_{\boldsymbol{x}}, \boldsymbol{x}$ records the variables which are $\tilde{\mu}$-bound to a computation on the path from the top of the term to the current position. $\langle x \| \star\rangle$ is in whnf, however it is not of the form $c_{x}$ since it demands variable $x$. Neither $\langle y \| \tilde{\mu} x . c\rangle$ nor $\langle\mu \star . c \| \tilde{\mu} x .\langle x \| \star\rangle\rangle$ are in whnf. A whnf is either of the form $C[\langle x \| F\rangle]$ or $C[\langle\lambda x . v \| \star\rangle]$. 
Proposition 3 (Unique Decomposition). A command $c$ is either a whnf or there exists a unique meta-context $C$ and redex $c^{\prime}$ such that $c$ is of the form $C\left[c^{\prime}\right]$.

Proposition 4 (Standardization). Given a command $c$ and a whnf $c^{\prime}$, if $c \rightarrow_{m l v} c^{\prime}$ then there exists a whnf $c^{\prime \prime}$ such that $c \mapsto_{m l v} c^{\prime \prime}$ and $c^{\prime \prime} \rightarrow_{m l v} c^{\prime}$.

\subsection{Soundness and Completeness of $\lambda_{m l v}$}

The $\lambda_{m l v}$ calculus is sound and complete with respect to the minimal restriction of the call-by-name sequent calculus, $\bar{\lambda} \mu \tilde{\mu}_{T}$. We first need to translate $\lambda_{m l v}$ terms to $\bar{\lambda} \mu \tilde{\mu}_{T}$ terms by giving a name to the $\tilde{\mu}$-term contained in a linear context. The only interesting cases of the translation, written as $(.)^{\circ}$ are:

$$
\begin{gathered}
\left(\left\langle v \| w_{1} \cdot \ldots w_{n} \cdot \alpha\right\rangle\right)^{\circ}=\left\langle v^{\circ} \| w_{1}^{\circ} \cdot \ldots w_{n}^{\circ} \cdot \alpha\right\rangle \\
\left(\left\langle v \| w_{1} \cdot \ldots w_{n} \cdot \tilde{\mu} x . c\right\rangle\right)^{\circ}=\left\langle\mu \star .\left\langle v^{\circ} \| w_{1}^{\circ} \cdot \ldots w_{n}^{\circ} \cdot \star\right\rangle \| \tilde{\mu} x . c^{\circ}\right\rangle
\end{gathered}
$$

we then have the following properties.

Proposition 5. (i) Given a $\lambda_{m l v}$ term $v, v={ }_{m l v} v^{\circ}$.

(ii) Given $\bar{\lambda} \mu \tilde{\mu}_{T}$ terms $v$ and $w$ :

(a) $v={ }_{m l v} w$ then $v=\bar{\lambda}_{\mu \tilde{\mu}_{T}} w$;

(b) $v=\bar{\lambda}_{\lambda \mu \tilde{\mu}_{T}}\langle\lambda x . w \| \star\rangle$ then $v={ }_{m l v} C\left[\left\langle\lambda x \cdot w^{\prime} \| \star\right\rangle\right]$ for some $C$ and $w^{\prime}$.

Indeed, $\lambda_{m l v}$ theory restricted to the call-by-name syntax of $\bar{\lambda} \mu \tilde{\mu}_{T}$ is included in $\bar{\lambda} \mu \tilde{\mu}_{T}$ theory.

Intermezzo 2 Soundness can also be shown with respect to the $\bar{\lambda} \mu \tilde{\mu}$ calculus without the need of doing a translation, since the $\bar{\lambda} \mu \tilde{\mu}$ calculus does not impose any restrictions on the context. This however requires extending the $\tilde{\mu}$ rule to $\left\langle v \| v_{1} \cdots v_{n} . \tilde{\mu} x . c\right\rangle \rightarrow c\left[x=\mu \star .\left\langle v \| v_{1} \cdots v_{n} . \star\right\rangle\right]$. The rule is sound for call-by-name extended with the eta rule, called $\eta_{\rightarrow}^{R}$ in [24], given as $y=\lambda x . \alpha .\langle y \| x . \alpha\rangle$.

\section{Minimal call-by-need in Natural Deduction $\left(\lambda_{\text {need }}\right)$}

We now present the call-by-need calculus inspired by the sequent calculus.

Definition 3. The syntax of $\lambda_{\text {need }}$ is defined as follows:

$$
\begin{array}{lll}
\text { term } & M & :=V \mid M_{n v} \\
\text { term value } & V & ::=x \| \lambda x . M \\
\text { computation } & M_{n v} & :=M M \mid \text { let } x=M \text { in } N \\
\text { applicative context } C_{a p} & ::=\square \mid C_{a p} M \\
\text { needed context } \quad C & ::=C_{a p} \mid \text { let } x=M_{n v} \text { in } C \mid \text { let } x=C_{a p} M \text { in } C[x]
\end{array}
$$

Reduction in $\lambda_{\text {need }}$, written as $\rightarrow_{\text {need }}$, is the compatible closure of the following rules:

$$
\begin{array}{lll}
(\beta) & (\lambda x . N) M & \rightarrow \text { let } x=M \text { in } N \\
(\text { lift }) & (\text { let } x=M \text { in } P) N & \rightarrow \text { let } x=M \text { in } P N \\
\left(\text { deref }_{v}\right) & \text { let } x=V \text { in } M & \rightarrow M[x \leftarrow V] \\
(\text { assoc }) & \text { let } z=(\text { let } x=M \text { in } N) \text { in } C[z] & \rightarrow \text { let } x=M \text { in let } z=N \text { in } C[z]
\end{array}
$$


The relation $\rightarrow{ }_{\text {need }}$ denotes the reflexive and transitive closure of $\rightarrow_{\text {need }}$.

The notion of weak head standard reduction is defined as:

$$
\frac{M \rightarrow_{\beta, \text { lift }} N}{C_{\beta l}[M] \mapsto_{\text {need }} C_{\beta l}[N]} \quad \frac{M \rightarrow_{\text {deref }_{v}, \text { assoc }} N}{C_{d a}[M] \mapsto_{\text {need }} C_{d a}[N]}
$$

where

$$
\begin{aligned}
& C_{\beta l}::=C_{a p} \| \text { let } x=M_{n v} \text { in } C_{\beta l} \| \text { let } x=C_{a p} \text { in } C[x] \\
& C_{d a}::=\square \| \text { let } x=M_{n v} \text { in } C_{d a}
\end{aligned}
$$

The notation $\mapsto_{\text {need }}$ stands for the reflexive and transitive closure of $\mapsto_{\text {need }}$. A weak head normal form (whnf) is a term $M$ such that for no $N, M \mapsto_{\text {need }} N$.

Unlike the calculi defined by Maraist et al. 26] and Ariola et al. [3], the $\operatorname{deref}_{v}$ rule follows the call-by-value discipline since it substitutes a value for each occurrence of the bound variable, even if the variable is not needed. The rule is derivable in the calculus of Maraist et al. using garbage collection. The assoc rule is more constrained than in the calculus of Maraist et al. since it performs the flattening of the bindings on a demand basis. The assoc requires the variable to appear in the hole of a context $C$, whose definition does not allow a hole to be bound to a let variable. For example, let $x=\square$ in $x$ and let $x=\square$ in let $y=x$ in $y$ are not $C$ contexts. This restriction is necessary to make sure that in a term of the form

$$
\text { let } x=(\text { let } z=N \text { in } P) \text { in let } y=x \text { in } y
$$

the standard redex is the substitution for $y$ and not the assoc redex. The assoc rule is more general than in 3 , since it does not require the binding for $z$ to be an answer (i.e., an abstraction surrounded by bindings). The lift rule is the same as in [26], it is more general than the corresponding rule in [3] since the left-hand side of the application is not restricted to be an answer. The calculi in [26] and [3] share the same standard reduction. For example, in the terms:

$$
\text { (let } y=M \text { in }(\lambda x . x) y) P \quad \text { let } y=(\text { let } z=N \text { in }(\lambda x . x) y) \text { in } y
$$

$(\lambda x . x) y$ is the standard redex. Our standard reduction differs. The above terms correspond to a lift and assoc redex, respectively. Moreover, our standard reduction is also defined for open terms. Thus, the following terms:

$$
\text { (let } y=x z \text { in } y) P \quad \text { let } y=(\text { let } z=x P \text { in } z) \text { in } y
$$

instead of being of the form $C[x]$, reduce further. The standard reduction requires different closure operations to avoid the interference between reductions. In

let $z=($ let $x=V$ in $N)$ in $z \quad$ let $y=($ let $z=($ let $x=M$ in $N)$ in $P)$ in $y$ the standard redex is the (outermost) assoc, and in let $x=I I$ in let $y=x$ in $y$, the $\operatorname{deref}_{v}$ is the standard redex.

Proposition 6. $\rightarrow_{\text {need }}$ is confluent. 
Proposition 7 (Unique Decomposition). A term $M$ is either a whnf or there exists a unique $C_{\beta l}$ such that $M$ is of the form $C_{\beta l}[P]$, where $P$ is a $\beta$ or lift redex, or there exists a unique $C_{d a}$ such that $M$ is of the form $C_{d a}[P]$, where $P$ is a deref $v$ or assoc redex.

The previous proposition essentially relies on the facts that $C[x]$ is a whnf and that $C_{d a} \subset C \subset C_{\beta l}$.

Proposition 8 (Standardization). Given a term $M$ and whnf $N$, if $M \rightarrow_{\text {need }} N$ then there exists a whnf $N^{\prime}$ such that $M \mapsto_{\text {need }} N^{\prime}$ and $N^{\prime} \rightarrow_{\text {need }} N$.

Definition 4. Let $\boldsymbol{x}$ be a sequence of variables. $M_{\boldsymbol{x}}$ is defined as: $M_{\boldsymbol{x}}::=\lambda x . N \mid$ let $y=N_{n v}$ in $M_{y \boldsymbol{x}}\left|z N_{1} \cdots N_{n}\right|$ let $y=z N N_{1} \cdots N_{n}$ in $C[y] \quad z \notin \boldsymbol{x}$

Proposition 9. A term $M$ is in whnf iff it is in $M_{\epsilon}$ (with $\epsilon$ the empty sequence).

\subsection{Soundness and completeness of $\lambda_{\text {need }}$}

Our calculus is sound and complete for evaluation to an answer (i.e., an abstraction or a let expression whose body is an answer) with respect to the standard reduction of the call-by-need calculi defined in [26] and [3], denoted by $\mapsto_{\text {mow }}^{a f}$.

Proposition 10. Let $M$ be a term and $A$ be an answer.

- If $M \mapsto_{\text {need }} A$ then there exists an answer $A^{\prime}$ such that $M \mapsto_{\text {mow }}^{a f} A^{\prime}$;

- If $M \mapsto{ }_{\text {mow }}^{a f} A$ then there exists an answer $A^{\prime}$ such that $M \mapsto_{n e e d} A^{\prime}$.

\section{Correspondence between $\lambda_{m l v}$ and $\lambda_{\text {need }}$}

The calculi $\lambda_{m l v}$ and $\lambda_{\text {need }}$ are in reduction correspondence for the following translations from $\lambda_{\text {need }}$ to $\lambda_{m l v}$ and vice-versa:

Definition 5. Given a term $M$ in $\lambda_{\text {need }}$, a term $v$, a context $e$ and a command $c$ in $\lambda_{\text {mlv }}$, translations $M^{\triangleright}, M_{e}^{\triangleright}, v^{\triangleleft}, e^{\triangleleft}$ and $c^{\triangleleft}$ are defined as follows:

$$
\begin{aligned}
& x^{\triangleright}=x \quad(\lambda x \cdot M)^{\triangleright}=\lambda x \cdot M^{\triangleright} \quad V_{e}^{\triangleright}=\left\langle V^{\triangleright} \| e\right\rangle \\
& \left.(M N)^{\triangleright}=\mu \star .(M N)_{\star}^{\triangleright} \quad(\text { let } x=M \text { in } N)^{\triangleright}=\mu \star \text {. (let } x=M \text { in } N\right)_{\star}^{\triangleright} \\
& (M N)_{e}^{\triangleright}=M_{N \triangleright \cdot e}^{\triangleright} \\
& \text { (let } x=M \text { in } N)_{e}^{\triangleright}= \begin{cases}M_{\tilde{\mu} x . N_{e}^{\triangleright}}^{\triangleright} & N \equiv C[x] \\
\left\langle M^{\triangleright} \| \tilde{\mu} x . N_{e}^{\triangleright}\right\rangle & \text { otherwise }\end{cases}
\end{aligned}
$$

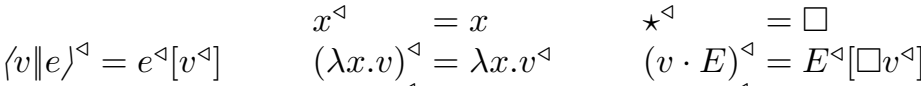

$$
\begin{aligned}
& (\mu \star . c)^{\triangleleft}=c^{\triangleleft} \quad(\tilde{\mu} x . c)^{\triangleleft}=\text { let } x=\square \text { in } c^{\triangleleft}
\end{aligned}
$$

We first illustrate the correspondence on an example. 
Example 3 Consider the following $\lambda_{\text {need }}$ reduction, where $I$ stands for $\lambda y \cdot y$ and $M$ for $(\lambda f . f I(f I))((\lambda z \cdot \lambda w . z w)(I I))$ :

$$
\begin{array}{ccc}
M \rightarrow_{\beta} \text { let } & \rightarrow_{\beta} \text { let } & \rightarrow \text { assoc let } \\
f=(\lambda z \cdot \lambda w . z w)(I I) & f=\text { let } & z=I I \\
\text { in } f I(f I) & z=I I & \text { in let } \\
& \text { in } \lambda w . z w & f=\lambda w . z w \\
& \text { in } f I(f I) & \text { in } f I(f I)
\end{array}
$$

We have $M_{\star}^{\triangleright}=\left\langle\lambda f \cdot \mu \star \cdot\left\langle f \| I \cdot(f I)^{\triangleright} \cdot \star\right\rangle \| \mu \star \cdot\left\langle\lambda z \cdot \lambda w \cdot(z w)^{\triangleright} \|(I I)^{\triangleright} \cdot \star\right\rangle \cdot \star\right\rangle$. The first $\beta$ step is simulated by the following $\lambda_{m l v}$ reduction, where we underline the redex to be contracted unless it occurs at the top:

$$
\begin{aligned}
& \left\langle\lambda f \cdot \mu \star \cdot\left\langle f \| I \cdot(f I)^{\triangleright} \cdot \star\right\rangle \| \mu \star \cdot\left\langle\lambda z \cdot \lambda w \cdot(z w)^{\triangleright} \|(I I)^{\triangleright} \cdot \star\right\rangle \cdot \star\right\rangle \rightarrow_{\beta} \\
& \left\langle\mu \star .\left\langle\lambda z \cdot \lambda w \cdot(z w)^{\triangleright} \|(I I)^{\triangleright} \cdot \star\right\rangle \| \tilde{\mu} f \cdot\left\langle\mu \star \star\left\langle f \| I \cdot(f I)^{\triangleright} \cdot \star\right\rangle \| \star\right\rangle\right\rangle \rightarrow \mu_{l} \\
& \left\langle\mu \star \cdot\left\langle\lambda z \cdot \lambda w \cdot(z w)^{\triangleright} \|(I I)^{\triangleright} \cdot \star\right\rangle \| \tilde{\mu} f \cdot\left\langle f \| I \cdot(f I)^{\triangleright} \cdot \star\right\rangle\right\rangle \rightarrow \mu_{l} \\
& \left\langle\lambda z \cdot \lambda w \cdot(z w)^{\triangleright} \|(I I)^{\triangleright} \cdot \tilde{\mu} f \cdot\left\langle f \| I \cdot(f I)^{\triangleright} \cdot \star\right\rangle\right\rangle
\end{aligned}
$$

The second $\mu_{l}$ step corresponds to moving the redex in the context let $f=$ $\square$ in $C[f]$ at the top. The simulation of the second $\beta$ step leads to:

$$
\left\langle(I I)^{\triangleright} \| \tilde{\mu} z \cdot\left\langle\lambda w \cdot(z w)^{\triangleright} \| \tilde{\mu} f \cdot\left\langle f \| I \cdot(f I)^{\triangleright} \cdot \star\right\rangle\right\rangle\right\rangle
$$

The assoc corresponds to an identity in $\lambda_{m l v}$.

Notice that the restriction on the assoc rule is embedded in the sequent calculus. The simulation of a non restricted assoc would require a generalization of the $\mu_{l}$ rule. For example, the simulation of the reduction:

$$
\text { let } x=(\text { let } y=I I \text { in } y) \text { in } 0 \rightarrow \text { let } y=I I \text { in let } x=y \text { in } 0
$$

would require equating the following terms:

$$
\langle\mu \star .\langle I \| I \cdot \tilde{\mu} y \cdot\langle y \| \star\rangle\rangle \| \tilde{\mu} x \cdot\langle 0 \| \star\rangle\rangle=\langle\mu \star \cdot\langle I \| I \cdot \star\rangle \| \tilde{\mu} y \cdot\langle y \| \tilde{\mu} x \cdot\langle 0 \| \star\rangle\rangle\rangle
$$

However, those should not be equated to $\langle I \| I \cdot \tilde{\mu} y \cdot\langle y \| \tilde{\mu} x \cdot\langle 0 \| \star\rangle\rangle \cdot \star\rangle$. That would correspond to relaxing the restriction of $E_{l}$ in the $\mu_{l}$ rule, and has the problem of bringing the redex $I I$ to the top and thus becoming the standard redex.

Proposition 11. Call-by-need reduction in natural deduction and sequent form are in reduction correspondence:

(i) $M \rightarrow{ }_{n e e d} M^{\triangleright \triangleleft}$

(ii) $c \rightarrow m l v c c^{\triangleleft \triangleright}$

(iii) If $M \rightarrow_{\text {need }} N$ then $M^{\triangleright} \rightarrow_{m l v} N^{\triangleright}$

(iv) If $c \rightarrow_{\text {mlv }} c^{\prime}$ then $c^{\triangleleft} \rightarrow_{\text {need }} c^{\prime \triangleleft}$

Remark 4 Note that the translation $\left({ }_{-}\right)_{e}^{\triangleright}$ of a let expression depends on the bound variable being needed or not. The choice of this optimized translation was required to preserve reduction. Indeed, otherwise, to simulate the assoc reduction one would need an expansion in addition to a reduction. 


\section{Classical call-by-need in sequent $\left(\lambda_{l v}\right)$ and natural deduction form $\left(\lambda \mu_{n e e d}\right)$}

Defining sequent classical call-by-need, called $\lambda_{l v}$, requires extending the applicative context and the $\mu$ construct to include a generic continuation variable ${ }^{2}$. The syntax of $\lambda_{l v}$ becomes:

$$
\begin{array}{ll}
c::=\langle v \| e\rangle & F::=\alpha \mid v \cdot E \\
C::=\square \|\langle\mu \alpha . c \| \tilde{\mu} z . C\rangle & E::=F \| \tilde{\mu} x . C[\langle x \| F\rangle] \\
v::=x \mid \lambda x . v \| \mu \alpha . c & e::=E \| \tilde{\mu} x . c
\end{array}
$$

Reduction, weak head standard reduction (written as $\rightarrow_{l v}$ and $\mapsto l v$, respectively) and weak head normal form (whnf) are defined as in the minimal case by replacing $\star$ with any context variable $\alpha$. For example, a term of the form $\langle\mu \alpha .\langle x \| \beta\rangle \| \tilde{\mu} x \cdot\langle y \| y \cdot \delta\rangle\rangle$ is in weak head normal form.

Unique decomposition, confluence and standardization extend to the classical case. Once control is added to the calculus, call-by-need and call-by-name are observationally distinguishable, as witnessed by the example given in the next section. It is important to notice that the bindings are not part of the captured context. For example, in the following command, the redex $I I$ written as $\mu \alpha .\langle\lambda x . x \|(\lambda x . x) \cdot \alpha\rangle$ will be executed only once. Whereas, if the bindings were part of the captured context then that computation would occur twice.

$$
\langle I I \| \tilde{\mu} z \cdot\langle\mu \alpha \cdot\langle\lambda x . \mu \beta \cdot\langle z \|(\mu \delta .\langle\lambda x . x \| \alpha\rangle) \cdot \beta\rangle \| \alpha\rangle \| \tilde{\mu} f \cdot\langle f \| z \cdot \gamma\rangle\rangle\rangle\rangle\rangle
$$

Unlike the sequent calculus setting, to extend minimal natural deduction to the classical case, we need to introduce two new constructs: the capture of a continuation and the invocation of it, written as $\mu \alpha . J$ and $[\alpha] M$, where $J$ stands for a jump (i.e., an invocation of a continuation). The reduction semantics makes use of the notion of structural substitution, which was first introduced in 30 and is written as $J[\alpha \leftarrow[\alpha] F]$ indicating that each occurrence of $[\alpha] M$ in $J$ is replaced by $[\alpha] F[M]$, where $F$ is the context captured by a continuation which is either $\square M$ or let $x=\square$ in $C[x]$. The benefits of structural substitution are discussed in [4]. In addition to lift, assoc, deref $v$ and $\beta$, the reduction theory includes the following reduction rules:

$$
\begin{array}{lll}
\left(\mu_{\text {ap }}\right) & (\mu \alpha . J) M & \rightarrow \mu \alpha . J[\alpha \leftarrow[\alpha](\square M)] \\
\left(\mu_{\text {let }}\right) & \text { let } x=\mu \alpha . J \text { in } C[x] & \rightarrow \mu \alpha . J[\alpha \leftarrow[\alpha](\text { let } x=\square \text { in } C[x])] \\
\left(\mu_{\text {lift }}\right) & \text { let } x=M_{n v} \text { in } \mu \alpha .[\beta] N & \rightarrow \mu \alpha .[\beta]\left(\text { let } x=M_{n v} \text { in } N\right) \\
\left(\mu_{\text {base }}\right) & {[\beta] \mu \alpha . J} & \rightarrow J[\alpha \leftarrow \beta]
\end{array}
$$

The relation $\rightarrow \mu_{\mu_{\text {need }}}$ denotes the compatible closure of $\rightarrow$, and $\rightarrow \mu_{\text {need }}$ denotes the reflexive and transitive closure of $\rightarrow \mu_{\text {need }}$. The weak head standard reduction is defined as follows:

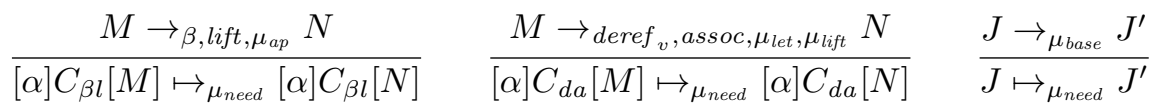

${ }^{2}$ To reduce closed commands one can introduce a constant named tp as in [5], or one can encode the top-level using subtraction (see Section 7). 
The notation $\mapsto \mu_{\text {need }}$ stands for the reflexive and transitive closure of $\mapsto \mu_{\text {need }}$. Note that we only reduce jumps. A weak head normal form (whnf) is a term $M$ such that for no $N, M \mapsto_{\mu_{\text {need }}} N$. For example, let $x=\mu \alpha$. $[\beta] P$ in $y x$ is in whnf.

Proposition 12. $\rightarrow_{\mu_{\text {need }}}$ is confluent.

Proposition 13. Given a term $M$ and whnf $N$, if $M \rightarrow \mu_{\text {need }} N$ then there exists a whnf $N^{\prime}$ such that $M \mapsto \mu_{\mu_{\text {need }}} N^{\prime}$ and $N^{\prime} \rightarrow_{\mu_{\text {need }}} N$.

The translation between classical call-by-need in natural and sequent form is modified in the following way to cover the classical constructs:

$$
\begin{aligned}
& (\mu \alpha . J)^{\triangleright}=\mu \alpha . J^{\triangleright} \quad([\alpha] M)^{\triangleright}=M_{\alpha}^{\triangleright} \\
& \begin{array}{cccc}
\alpha_{\alpha} & =\alpha & F_{\alpha} & =\square
\end{array} \\
& \alpha_{v \cdot E} \quad=\alpha_{E} \quad F_{v \cdot E}=F_{E}\left[\square v^{\triangleleft}\right] \\
& \alpha_{\tilde{\mu} x .\langle v \| e\rangle}=\alpha_{e} \quad F_{\tilde{\mu} x .\langle v \| e\rangle}=\text { let } x=\square \text { in } F_{e}\left[v^{\triangleleft}\right] \\
& \mu \alpha . c^{\triangleleft}=\mu \alpha \cdot c^{\triangleleft} \quad e^{\triangleleft}=\left[\alpha_{e}\right] F_{e}
\end{aligned}
$$

Proposition 14. Classical call-by-need in natural deduction and sequent form are in equational correspondence:

(i) $M=\mu_{\text {need }} M^{\triangleright \triangleleft}$

(ii) $c=l_{l v} c^{\triangleleft \triangleright}$

(iii) If $M={ }_{\mu_{\text {need }}} N$ then $M^{\triangleright}={ }_{l v} N^{\triangleright}$

(iv) If $c={ }_{l v} c^{\prime}$ then $c^{\triangleleft}={ }_{\mu_{\text {need }}} c^{\prime \triangleleft}$

Notice that the main reason for having only equational correspondence instead of a more precise reduction correspondence is the fact that, in $\lambda \mu_{\text {need }}, \mu_{a p}$ can be applied atomically $(\mu \alpha . J) N_{1} \ldots N_{n} \rightarrow_{\mu_{a p}}\left(\mu \alpha . J\left[\alpha \leftarrow[\alpha] \square N_{1}\right]\right) N_{2} \ldots N_{n}$ while in $\lambda_{l v}$ the whole applicative context $\square N_{1} \ldots N_{n}$ is moved at once. In particular the following reduction still holds $c \rightarrow_{l v} c^{\prime}$ then $c^{\triangleleft} \rightarrow \mu_{\text {need }} c^{\triangleleft}$.

\section{Dual classical call-by-need in sequent form $\left(\lambda_{l n}\right)$}

In call-by-need, the focus is on the consumer and goes to the producer on a need basis. This suggests a dual call-by-need which corresponds to focusing on the producer and going to the consumer on a need basis. To that end, we first extend the classical call-by-need calculus of the previous section, $\lambda_{l v}$, with the dual of the implication, the subtraction connective, and then build the dual classical call-by-need calculus by using duality constructions typical from $\bar{\lambda} \mu \tilde{\mu}$-calculi.

While $\mu$ and $\tilde{\mu}$ constructs are dual of each other, implicative constructions $\lambda x$.t and $t \cdot E$ currently have no dual in $\lambda_{l v}$. We extend $\lambda_{l v}$ by adding constructions for the subtraction connective [11. Subtraction was already considered in the setting of $\bar{\lambda} \mu \tilde{\mu}$ in Curien et al. [12. We follow the notation introduced by Herbelin in his habilitation thesis [24]. Terms are extended with the construction $v-e$ and contexts with $\tilde{\lambda} \alpha . e$. The corresponding reduction is:

$$
(-) \quad\left\langle v-e \| \tilde{\lambda} \alpha \cdot e^{\prime}\right\rangle \rightarrow\left\langle\mu \alpha \cdot\left\langle v \| e^{\prime}\right\rangle \| e\right\rangle
$$


We can now present the classical call-by-need calculus extended with subtraction, $\lambda_{l v}^{-}$. The structural rules are obtained by instantiating $\mathcal{V}$ and $\mathcal{E}$ as:

$V_{v}^{-}=x\|\lambda x . t\|\left(V_{v}^{-}-e\right) \quad E_{l}^{-}=F^{-} \| \tilde{\mu} x . C_{l}^{\tilde{\mu}}\left[\left\langle x \| F^{-}\right\rangle\right]$with $F^{-}=\alpha \mid v \cdot E_{l}^{-} \| \tilde{\lambda} \alpha . e$ The syntax for the language with subtraction is finally as follows (with $c=\langle v \| e\rangle$ ): meta-context $C::=\square \mid\langle\mu \alpha . c \| \tilde{\mu} x . C\rangle \quad$ applicative context $F::=\alpha \mid v \cdot E \| \tilde{\lambda} \alpha . e$ linear term $\quad V::=x|V-e| \lambda x . v \quad$ linear context $\quad E::=F \mid \tilde{\mu} x \cdot C[\langle x \| F\rangle]$ term $\quad v::=V \mid \mu \alpha . c \quad$ context $\quad e:=E \| \tilde{\mu} x . c$

Using the duality principles developed in [12, we obtain $\lambda_{l n}^{-}$by dualizing $\lambda_{l v}^{-}$: The syntax of the calculus is obtained by dualizing $\lambda_{l v}^{-}$syntax and its reductions are also obtained by duality: $(\beta)$ and $(-)$ are dual of each other while $\mu_{v}$ and $\tilde{\mu}_{l}$ are respectively turned into:

- the $\mu$-reduction associated with set $E_{n}^{-}::=\alpha\left|t \cdot E_{n}^{-}\right| \tilde{\lambda} \alpha . e$, written $\mu_{n}$

- the $\tilde{\mu}$-reduction associated with set $V_{l}^{-}::=W \| \mu \alpha . C_{l}^{\mu}[\langle W \| \alpha\rangle]$, with $W::=$ $x|\lambda x . t| W-e$ (and $C_{l}^{\mu}$ being the dual of $C_{l}^{\tilde{\mu}}$ ), written $\tilde{\mu}_{l}$.

Since only linear contexts are substituted for context variables, as in call-byname, but only on a needed basis, we call the resulting calculus lazy call-by-name. Its syntax is given as follows:

$$
\begin{array}{lll}
\text { meta-context } C::=\square \|\langle\mu \alpha . C \| \tilde{\mu} x . c\rangle & \text { linear term } & W::=x|\lambda x . t| W-e \\
\text { linear context } E::=\alpha|\tilde{\lambda} \alpha . e| t \cdot E & \text { value } & V::=W \mid \mu \alpha . C[\langle W \| \alpha\rangle] \\
\text { context } \quad e::=E \mid \tilde{\mu} x . c & \text { term } & t::=V \| \mu \alpha . c
\end{array}
$$

The four theories can be discriminated by the following command:

$$
\begin{gathered}
c \equiv\left\langle\mu \alpha .\left\langle\lambda x . \mu \_.\langle\lambda y \cdot x \| \alpha\rangle \| \alpha\right\rangle\right. \\
\| \tilde{\mu} f \cdot\langle\mu \beta .\langle f \| t \cdot \beta\rangle \\
\| \tilde{\mu} x_{1} \cdot\langle\mu \gamma \cdot\langle f \| s \cdot \gamma\rangle \\
\left.\left.\left.\| \tilde{\mu} x_{2} \cdot\left\langle x_{1} \| x_{2} \cdot x_{2} \cdot \delta\right\rangle\right\rangle\right\rangle\right\rangle
\end{gathered}
$$

We call $c_{1}$ the command obtained by instantiating $t$ and $s$ to $\lambda x . \lambda y . x$ and $\lambda x . \lambda y . y$, respectively. Then $c_{1}$ evaluates to $\langle\lambda x . \lambda y . x \| \delta\rangle$ in lazy call-by-value and to $\langle\lambda x . \lambda y . y \| \delta\rangle$ in call-by-name. We call $c_{2}$ the command obtained by instantiating $t$ and $s$ to $\lambda f \cdot \lambda x \cdot \mu \alpha \cdot\langle f \| x \cdot \alpha\rangle$ and $\lambda x . x$. We now consider $c_{3}$ to be $\left\langle\mu \gamma \cdot c_{2} \| \tilde{\mu} w . c_{1}\right\rangle$, where $w$ does not occur free in $c_{1}$ and $\gamma$ does no occur free in $c_{2}$. In call-by-name and lazy call-by-value, $c_{3}$ evaluates as $c_{1}$, up to garbage collection. However, $c_{3}$ evaluates to $\langle\lambda f . \lambda x . \mu \alpha .\langle f \| x \cdot \alpha\rangle \| \delta\rangle$ in call-by-value, and to $\langle I \| \delta\rangle$ in lazy call-by-name, up to garbage collection. This can be generalized by the following example, where we assume that $\alpha_{1}$ does not occur free in $c$ and $V$, and that $x_{1}$ does not occur free in $c^{\prime}$ and $E$. If we define

$$
\begin{aligned}
c_{0} \triangleq\left\langle\mu \alpha_{1} \cdot\left\langle\mu \alpha_{2} \cdot\left\langle V \| \alpha_{2}\right\rangle \| \tilde{\mu} y . c\right\rangle \| \tilde{\mu} x_{1} \cdot\left\langle\mu \beta . c^{\prime} \| \tilde{\mu} x_{2} \cdot\left\langle x_{2} \| E\right\rangle\right\rangle\right\rangle \\
\text { then } \quad c_{0} \rightarrow_{n} c^{\prime}\left[\beta \leftarrow E\left[x_{2} \leftarrow \mu \beta . c^{\prime}\right]\right] \\
c_{0} \rightarrow_{v} c\left[y \leftarrow V\left[\alpha_{2} \leftarrow \tilde{\mu} y \cdot c\right]\right] \\
c_{0} \rightarrow_{l n}\left\langle\mu \alpha_{1} \cdot c\left[y \leftarrow \mu \alpha_{2} \cdot\left\langle V \| \alpha_{2}\right\rangle\right] \| \tilde{\mu} x_{1} \cdot\left\langle\mu \beta . c^{\prime} \| \tilde{\mu} x_{2} \cdot\left\langle x_{2} \| E\right\rangle\right\rangle\right\rangle \\
c_{0} \rightarrow_{l v}\left\langle\mu \alpha_{1} \cdot\left\langle\mu \alpha_{2} \cdot\left\langle V \| \alpha_{2}\right\rangle \| \tilde{\mu} y \cdot c\right\rangle \| \tilde{\mu} x_{1} \cdot c^{\prime}\left[\beta \leftarrow \tilde{\mu} x_{2} \cdot\left\langle x_{2} \| E\right\rangle\right]\right\rangle
\end{aligned}
$$




\section{Conclusions and Future work}

The advantage of studying evaluation order in the context of sequent calculus has shown its benefits: extending the calculus (both syntax and reduction theory) to the classical case simply corresponds to going from one context variable to many. The study has also suggested how to provide a call-by-need version of Parigot's $\lambda \mu$-calculus, and in the minimal case, has led to a new notion of standard reduction, which applies the lift and assoc rule eagerly. In the minimal case, the single context variable, called $\star$, could be seen as the constant tp discussed in 615. In the cited work, it is also presented how delimited control can be captured by extending tp to a dynamic variable named $\widehat{t p}$. This suggests that one could use $\widehat{t p}$ instead of $\mathbf{t p}$ to represent computations also in the minimal setting. Since evaluation goes under a $\widehat{t p}$, it means that one would obtain a different notion of standard reduction, which would correspond to the one of Ariola et al. [3] and Maraist et al. [26].

A benefit of sequent calculus over natural deduction in both call-by-name and call-by-value is that the standard redex in the sequent calculus always occurs at the top of the command. In other words, there is no need to perform an unbounded search to reach the standard redex 2]: this search is embedded in the structural reduction rules. However, this does not apply to our call-by-need sequent calculus: the standard redex can be buried under an arbitrary number of bindings. This can be easily solved by considering a calculus with explicit substitutions. A command now becomes $\langle v \| e\rangle \boldsymbol{\tau}$, where $\boldsymbol{\tau}$ is a list of declarations. For example, the critical pair will be solved as: $\left\langle\mu \alpha . c \| \tilde{\mu} x . c^{\prime}\right\rangle \boldsymbol{\tau} \rightarrow c^{\prime}[x=\mu \alpha . c] \boldsymbol{\tau}$ and the switching of context is realized by the rule: $\langle x \| E\rangle \boldsymbol{\tau}_{\mathbf{0}}[x:=\mu \alpha . c] \boldsymbol{\tau}_{\mathbf{1}} \rightarrow$ $c\left[\alpha:=\tilde{\mu} x .\langle x \| E\rangle \boldsymbol{\tau}_{\mathbf{0}}\right] \boldsymbol{\tau}_{\mathbf{1}}$. This will naturally lead us to developing abstract machines, which will be compared to the abstract machines of Garcia et al. [22] and Danvy et al. [15], inspired by natural deduction.

We have related the lazy call-by-value with subtraction to its dual. We plan to provide a simulation of lazy call-by-value in lazy call-by-name and vice-versa, without the use of subtraction. We are also interested in devising a complete set of axioms with respect to a classical extension of the call-by-need continuationpassing style of Okasaki et al. [29]. A natural development will then be to extend our lazy call-by-value and lazy call-by-name with delimited control. Following a suggestion by Danvy, we will investigate connections between our lazy callby-name calculus and a calculus with futures [28. At last, we want to better understand the underlying logic or type system.

\section{References}

1. Z. Ariola and H. Herbelin. Minimal classical logic and control operators. In ICALP'03, volume 2719 of Lecture Notes in Computer Science. Springer, 2003.

2. Z. M. Ariola, A. Bohannon, and A. Sabry. Sequent calculi and abstract machines. ACM TOPLAS, 31(4):275-317, 2009.

3. Z. M. Ariola and M. Felleisen. The call-by-need lambda calculus. JFP, 1997.

4. Z. M. Ariola and H. Herbelin. Control reduction theories: the benefit of structural substitution. J. Funct. Program., 18(3):373-419, 2008. 
5. Z. M. Ariola, H. Herbelin, and A. Sabry. A proof-theoretic foundation of abortive continuations. Higher Order Symbol. Comput., 20:403-429, December 2007.

6. Z. M. Ariola, H. Herbelin, and A. Sabry. A type-theoretic foundation of delimited continuations. Higher Order Symbol. Comput., 22:233-273, September 2009.

7. Z. M. Ariola, H. Herbelin, and A. Saurin. Classical call-by-need and duality, extended version. www.pps.jussieu.fr/ ${ }^{\sim}$ saurin/Publi/need_duality_long.pdf

8. H. Barendregt and S. Ghilezan. Lambda terms for natural deduction, sequent calculus and cut elimination. J. Funct. Program., 10:121-134, January 2000.

9. H. P. Barendregt. The Lambda Calculus: Its Syntax and Semantics. 1984.

10. A. Church. A set of postulates for the foundation of logic. Annals of Math., 1932.

11. T. Crolard. Subtractive Logic. TCS, 254(1-2):151-185, 2001.

12. P.-L. Curien and H. Herbelin. The duality of computation. In ICFP, 2000.

13. V. Danos, J.-B. Joinet, and H. Schellinx. LKQ and LKT: sequent calculi for second order logic based upon dual linear decompositions of the classical implication. In Advances in Linear Logic, volume 222, pages 211-224. CUP, 1995.

14. V. Danos, J.-B. Joinet, and H. Schellinx. A new deconstructive logic: Linear logic. J. Symb. Log., 62(3):755-807, 1997.

15. O. Danvy, K. Millikin, J. Munk, and I. Zerny. Defuntionalized interpreters for callby-need evaluation. In Functional and Logic Programming, FLOPS2010, 2010.

16. R. David and W. Py. Lambda-mu-calculus and Böhm's theorem. JSL, 2001.

17. J. H. Fasel, P. Hudak, S. Peyton Jones, and P. W. (editors). Haskell special issue. SIGPLAN Notices, 27(5), May 1992.

18. M. Felleisen and D. Friedman. Control operators, the SECD machine, and the lambda-calculus. In Formal description of programming concepts-III, 1986.

19. M. Felleisen, D. Friedman, and E. Kohlbecker. A syntactic theory of sequential control. Theoretical Computer Science, 52(3):205-237, 1987.

20. M. Felleisen, D. Friedman, E. Kohlbecker, and B. Duba. Reasoning with continuations. In LICS, pages 131-141, 1986.

21. A. Filinski. Declarative Continuations and Categorical Duality. Ms thesis, 1989.

22. R. Garcia, A. Lumsdaine, and A. Sabry. Lazy evaluation and delimited control. In POPL. ACM, 2009.

23. G. Gentzen. Investigations into logical deduction. In M. Szabo, editor, Collected papers of Gerhard Gentzen, pages 68-131. North-Holland, 1969.

24. H. Herbelin. C'est maintenant qu'on calcule. In Thèse d'habilitation, 2005.

25. H. Herbelin and S. Zimmermann. An operational account of call-by-value minimal and classical $\lambda$-calculus in "natural deduction" form. In TLCA, LNCS, 2009.

26. J. Maraist, M. Odersky, and P. Wadler. The call-by-need $\lambda$-calculus. JFP, 1998.

27. E. Moggi. Computational $\lambda$-calculus and monads. In LICS, 1989.

28. J. Niehren, J. Schwinghammer, and G. Smolka. A concurrent lambda calculus with futures. Theor. Comput. Sci., 364:338-356, November 2006.

29. C. Okasaki, P. Lee, and D. Tarditi. Call-by-need and continuation-passing style. In Lisp and Symbolic Computation, pages 57-81. Kluwer Academic Publishers, 1993.

30. M. Parigot. Lambda-mu-calculus: An algorithmic interpretation of classical natural deduction. In LPAR 92, pages 190-201. Springer-Verlag, 1992.

31. G. D. Plotkin. Call-by-name, call-by-value and the lambda-calculus. TCS, 1, 1975.

32. D. Prawitz. Natural Deduction, a Proof-Theoretical Study. 1965.

33. S. R. Della Rocca and L. Paolini. The Parametric $\lambda$-Calculus. Springer, 2004.

34. A. Sabry and M. Felleisen. Reasoning about programs in continuation-passing style. Lisp and Symbolic Computation, 6(3-4):289-360, 1993.

35. A. Saurin. Separation with streams in the $\Lambda \mu$-calculus. In LICS. IEEE, 2005.

36. P. Selinger. Control categories and duality: on the categorical semantics of the lambda-mu calculus. Math. Struct. in Comp. Science, 11(2):207-260, 2001. 